\title{
Prescription opioid abuse: pharmacists' perspective and response
}

This article was published in the following Dove Press journal:

Integrated Pharmacy Research and Practice

25 August 2016

Number of times this article has been viewed

\author{
Gerald Cochran ${ }^{1,2}$ \\ Valerie Hruschak ${ }^{2}$ \\ Brooke DeFosse ${ }^{3}$ \\ Kenneth C Hohmeier ${ }^{3}$ \\ 'Department of Psychiatry, School \\ of Medicine, ${ }^{2}$ School of Social Work, \\ University of Pittsburgh, Pittsburgh, \\ PA, ${ }^{3}$ Department of Clinical Pharmacy, \\ College of Pharmacy, University of \\ Tennessee, Memphis, TN, USA
}

\begin{abstract}
Opioid medication abuse and overdose are major concerns for public health, and a number of responses to address these issues have taken place across the US. Pharmacists and the pharmacy profession have made important contributions as a part of the response to this national crisis. This article provides a brief review of the antecedents, driving forces, and health status of patients involved in the opioid medication and overdose epidemic. This review further discusses pharmacy-based actions that have been undertaken to address this issue, including prescription drug monitoring, take-back, and naloxone training/distribution programs. This review likewise examines current efforts underway in the field to educate practitioners and needed future steps that must be taken by pharmacists in order to continue the profession's pivotal role in working toward resolving this national public health problem. In particular, evidence and arguments are presented for proactively identifying and intervening with patients who abuse and/or are at risk for overdose. Continued and active engagement by pharmacists in these efforts has the potential to result in important reductions in opioid medication abuse and overdose and improvements for patient's health.
\end{abstract}

Keywords: opioid pain medication, addiction, pharmacy practice

\section{Introduction}

Trends in the nonmedical use of prescription opioids (NMPO), related health problems, and opioid medication overdose mortality in the US have reached epidemic proportions. ${ }^{1}$ These challenges currently facing the nation require a multifaceted approach to achieve resolution. A number of policy- and clinical-level actions have been employed to combat this issue. The pharmacy profession has been an integral part in taking both direct and indirect steps to confront NMPO and overdose. This article provides a brief review of the opioid medication epidemic, pharmacy-based efforts to address this issue, and needed future steps that must be taken by pharmacists and the pharmacy profession must act upon as they continue to play a pivotal role working toward resolving this national public health crisis. We recognize that within the empirical literature, there are a number of conceptual/operational definitions and behaviors that characterize problematic consumption of opioid medications. ${ }^{2,3}$ In this review, we categorize these definitions and behaviors as NMPO for the purpose of simplicity.

\section{Opioid medication epidemic and epidemiology}

Opioids are one of the primary drugs of abuse in the world. ${ }^{4}$ An estimated 26-36 million individuals use opioids illicitly internationally, with approximately one-half to one-third
Correspondence: Gerald Cochran Department of Psychiatry, School of Medicine, University of Pittsburgh, 4200 Forbes Avenue \#2006, Pittsburgh, PA 15260, USA

Tel + I 4126242325

Fax + I 4I26246323

Email gcochran@pitt.edu 
of illicit opioid use being nonheroin consumption. ${ }^{4}$ The US is the largest consumer of prescription opioid medication compared to every other nation in the world. ${ }^{5}$ Data indicate that Americans consume $>80 \%$ of the world's prescription opioids, regardless of the fact that the US comprises $<5 \%$ of the world's population. ${ }^{6}$ Opioid medication sales in the US have increased 150\% between 1997 and 2007, with the average milligrams of opioids used per person increasing at the same time $>400 \% .^{7}$ A large contributor to this rapid uptake in opioid medication consumption was the inclusion of pain as the "fifth vital sign" during the 1990s. ${ }^{8}$ The inclusion of pain as the fifth vital sign stemmed from the American Pain Society's efforts to elevate awareness among health care professionals regarding pain treatment and was intended to improve attention to pain management nationally. ${ }^{8}$ During this same time in the US, opioid pain medications, such as OxyContin, were heavily marketed for nonmalignant pain. ${ }^{9}$ This marketing campaign included claims by manufacturers that there was little risk of addiction to opioid analgesics. ${ }^{9}$ Moreover, targeted sales marketing accompanied by large cash bonuses to pharmaceutical sales representatives occurred for promoting and increasing the distribution of these medications, particularly OxyContin. ${ }^{9}$ Despite claims of limited risk for addiction, an additional instrumental factor motivating this epidemic is the abuse liability of opioid medications. Users of opioid pain medications report that these drugs can produce a heroin-like euphoria or "high". In addition to psychoactive properties, street values for these medications approximate or are greater than heroin. ${ }^{10}$ Regular consumption of these medications can produce physical dependence. Consequently, withdrawal symptomology, which includes vomiting, diarrhea, nausea, chills, cramps, and increased blood pressure, can increase the likelihood for continued consumption. ${ }^{11}$

The total number of persons in 2012 who reported NMPO was 4.9 million, second to only those using marijuana at 18.9 million. ${ }^{12}$ Among those with NMPO, 2.1 million individuals were diagnosed as having abused or were dependent on opioid pain medications. ${ }^{12}$ Annual societal costs in the US stemming from NMPO have been calculated to be $\sim \$ 56$ billion. ${ }^{13}$ Half of those reporting NMPO obtain medications from friends or relatives who had the prescriptions under their possession. ${ }^{12}$ Additionally, one-fifth of individuals engaged in NMPO obtain opioid medications through "shopping" behaviors, ${ }^{12}$ wherein patients visit multiple doctors and pharmacies seeking prescriptions and fills. ${ }^{14,15}$

New trends in street opioid use indicate unintended consequences in addressing NMPO. Emerging research points to NMPO as a likely starting point for heroin use. In a study from 2002 to 2011, heroin use reported by respondents in the previous 12 months was 19 times greater for those who had previously engaged in NMPO ${ }^{16}$ This transition from NMPO to heroin use has been consistently documented across a number of studies, and while all patients who engage in NMPO do not initiate heroin use, evidence supports this graduated trend in substance use serverity. ${ }^{17,18}$

Individuals engaged in NMPO have been noted to suffer from a number of health comorbidities. In specific terms, those engaged in NMPO are more likely to have additional mental and behavioral health conditions, ${ }^{19-22}$ including posttraumatic stress, mood, anxiety, personality, ${ }^{19,21,23-25}$ and substance use disorders. ${ }^{19-22,26,27}$ Common comorbid health problems among those engaged in NMPO include pain conditions, ${ }^{20,22,25,28,29}$ hepatitis, ${ }^{19,26}$ and overall poorer health. ${ }^{21,29}$ Heavy users of prescription opioid medications have also been found to have disproportionately higher rates of HIV infection, ${ }^{30}$ immunosuppression, ${ }^{31}$ hypertension, stomach ulcers, and urination/bladder problems. ${ }^{29}$ Another aspect of chronic and heavy opioid usage is structural and functional changes in regions of the brain that can impact regulation of affect, impulse control, and reward and motivational functions. $^{32}$ The most detrimental consequence of the NMPO epidemic has been the associated overdose deaths, which increased fourfold from 1999 to $2008,{ }^{33}$ with 44 individuals per day currently dying of fatal overdoses. ${ }^{34}$ Altogether, deaths in 2010 involving prescription opioid painkillers $(\mathrm{N}=16,651)$ accounted for $45 \%$ of all illicit and prescription drug overdose deaths combined. ${ }^{35}$ Given the impact of NMPO in the US, a broad-based concerted response is necessary.

\section{Efforts to address NMPO and overdose Prescription drug monitoring programs}

Responses to the national NMPO epidemic in the US have spanned the continuum of social and health care arenas and include, for example, activities of law enforcement, ${ }^{36}$ government, ${ }^{17}$ pharmaceutical companies,,${ }^{37,38}$ and clinical health care providers. ${ }^{39}$ One of the furthest reaching efforts in the US has been a policy solution designed to impact prescribing and patient access to medications. Prescription drug monitoring programs (PDMPs) are large scale data collection, reporting efforts implemented by states to supply prescribers and pharmacists with information regarding prescribing patterns and medication fills in order to help identify drug-seeking patients or those with inappropriate prescriptions. ${ }^{40}$ With the exception of Missouri, PDMPs have been implemented in varying degrees in each of the US states. ${ }^{41}$ 
Possessing data indicating questionable drug-seeking and/ or use patterns among patients, prescribers, and pharmacists can assist in the better management of writing and filling prescriptions for those who might be engaged in NMPO. ${ }^{40,41}$ The effectiveness of PDMPs, however, has been evaluated in empirical studies and has shown mixed outcomes, underutilization, ${ }^{40}$ and limited effectiveness in reducing consumption and overdose deaths. ${ }^{42}$ Furthermore, PDMPs have also been noted to operate within limited environments for patient identification and data sharing. ${ }^{43}$ Research data likewise show that PDMPs have limited use among some practitioners primarily because of time restraints, difficulty in accessing and navigating systems, and beliefs that using the system will not change patients' behaviors. ${ }^{44}$ Evidence also exists showing that pharmacists feel discomfort in assuming a "policing" role, wherein they are expected to monitor and confront patients. ${ }^{45-47}$ In addition, what is not apparent in the literature regarding PDMP is their effects on NMPO among patients for actual improvement in health.

Potentially valuable additions to PDMPs would ideally include more extensive data collection related to patients' demographic and health information within these systems in order to provide richer profiles of patients' health, medication usage, and demographic characteristics. For instance, connecting these programs with patient's health information collected in electronic health records could add valuable information regarding patient's risk for NPMO. Additionally, linking narcotic medication prescriptions in PDMPs with other medications filled by patients could yield important results for pharmacists to know if patients have concomitant fills for mental health medications, muscle relaxants, or benzodiazepines. Similarly, improvements could result from linking these systems with prescription dispensing software to better integrate PDMP processes into the established pharmacy workflow.

While possessing information regarding patient's medication use is valuable for pharmacists, PDMPs could be further enhanced if they were to also produce tips or guidance for intervention purposes. For example, if a patient is seeking an early refill, pharmacists would not only be alerted to such behaviors by the PDMP system but also ideally could be given action steps for initiating a targeted medication therapy management (MTM) session. ${ }^{48}$ This session would be aimed at helping patients increase adherence to their prescribed medication regimen. Such targeted MTM sessions have demonstrated efficacy for the improvement of medication adherence and health problems, including alcohol problems and bone density screening and education. ${ }^{48-51}$ Furthermore, pharmacists presented with at-risk patients could also be instructed from within the system to not only consult prescribers of the medications being filled, but instructions could also be given to pharmacists to refer patients (including the system providing up-to-date contact information) to additional needed health services, such as mental health counseling, health coaching, and social work services available from insurers. ${ }^{45}$

\section{Take-back programs}

Other efforts to reduce NMPO that have involved pharmacy are drug take-back programs across the US. ${ }^{52-54}$ These programs typically operate in conjunction with local law enforcement entities and involve widespread advertising in target areas. These efforts solicit individuals to "drop off" unused or excess medications without punitive or legal repercussions. Medications are dropped off by community members to law enforcement officials in pharmacy parking lots or are deposited in secure, unmanned containers within commercial pharmacy settings. Given that patients who receive medications may discard drugs (eg, in the trash and sewer) or retain them after discontinuing use, ${ }^{52}$ take-back programs are designed to prevent inappropriate disposal for environmental protection and to prevent used medications from being stolen or consumed by those to whom they were not prescribed. This is especially valuable given that, as mentioned earlier, a large portion of medication diversion occurs from friends or relatives who had the prescriptions under their possession. ${ }^{12}$

Community members generally have encouraging views of take-back programs, ${ }^{54}$ and those who have participated in these events have also reported positive experiences when dropping off medications. ${ }^{52,54}$ These programs have reported limited success for addressing NMPO in that they have been documented to have taken-back mostly nonnarcotic drugs. ${ }^{53,55}$ Measurement of outcomes from take-back programs regarding impact on individuals has also been narrow in terms of the impact on patient-level and health improvements or reductions in overdose within communities. More detailed research on these programs and their impact on NMPO are needed.

While the anonymity and ease of take-back programs is certainly beneficial, the high number of individuals utilizing this resource could be viewed as an opportunity for patient screening, intervention, and possible referral to ancillary health services. Take-back programs occurring within community pharmacy locations could be an excellent venue for pharmacists and/or pharmacy staff members to attempt engaging patients regarding their health to assess for NMPO 
or explore with patients' additional health problems that could put them at-risk for future issues with their opioid medications. If patients are identified as at-risk or engaged in NMPO, pharmacists could work to assist these individuals to receive appropriate information to safeguard their health or resources to receive additional health services.

\section{Naloxone training and distribution}

Another major effort targeting this national epidemic focuses specifically on addressing overdose. Rapidly escalating overdose deaths has called national attention to the need for effective approaches to reach individuals at-risk of overdose from illicit heroin use and NMPO. ${ }^{56}$ While overdose previously has been in large part associated with injection drug use, trends over the past decade regarding NMPO indicate overdose risk is not restricted to heroin users. Overdose risk is particularly pertinent to those who consume high doses of opioids for pain management and/or those who mix opioid medications with other psychoactive substances. ${ }^{57,58}$ Given these issues, there has been a growing body of evidence to support community-level approaches to prevent opioid overdose deaths through various harm reduction strategies. Such efforts have focused training on how to respond effectively to opioid overdose through community-based overdose education and naloxone distribution (OEND) programs. OEND programs have concentrated their efforts on individuals using opioid medications and bystanders who are at an increased likelihood to witness an opioid overdose. ${ }^{59}$ The content of OEND programs has mainly centered on how to respond appropriately to overdose including the emergency administration of Naloxone, an opioid antidote to revive individuals experiencing an overdose. ${ }^{60}$

Naloxone is not considered a controlled substance as it has no potential for abuse, and it has been employed by medical professionals in the US for $>40$ years as the bestpractice for opioid overdose resuscitation. ${ }^{61}$ In the early 1990s, health professionals first called for the provision of naloxone outside of inpatient settings. ${ }^{59}$ The first programs in the US to dispense naloxone for fatal overdose prevention were in the early 1990s, and by the mid-2000s, OEND programs were scattered across the US. ${ }^{62}$ As of June 2014, 644 community-based OEND programs were in operation in the US, and participants reported reversing $>26,463$ overdose events. ${ }^{63}$ Research data support the effectiveness of OEND. An analysis of OEND programs conducted between 2006 and 2009 in Massachusetts communities with high opioid overdose rates demonstrated a significant reduction in overdose mortality within those communities that implemented these programs. ${ }^{64}$ Furthermore, researchers have determined that naloxone distribution programs are cost effective. ${ }^{65}$

Despite these advancements in having patient access to naloxone, the US Food and Drug Administration has expressed minimal interest in supporting these efforts. ${ }^{59}$ As an alternative, a number of states have adapted Collaborative Pharmacy Practice Agreements (CPAs). CPAs for naloxone involve prescribers authorizing pharmacists to dispense naloxone to patients in the pharmacy setting without patients needing to visit prescribers in person. ${ }^{59}$ CPAs utilize pharmacists as drug therapy experts and ultimately are intended to improve the efficiency and quality of patient care. ${ }^{59}$ As of 2014 , collaborative practice authority existed in 48 states. However, wide variation exists between states regarding regulations of what authority can be delegated from prescribers to pharmacists under these agreements. ${ }^{59}$ In addition to CPAs, other states (such as California, New Mexico, and Vermont) have adopted statewide protocols for naloxone prescribing in pharmacies. ${ }^{66}$ These dispensing protocols are between the state and pharmacists and are employed when states have significant interest in improving public health via pharmacist prescribing. While naloxone distribution is not necessarily a sole solution to NMPO and related overdose, it is a crucial adjunct to other measures for addressing this national problem. ${ }^{67}$

\section{Response of professional pharmacy organizations}

One of the key national pharmacy organizations supporting naloxone distribution and education has been the American Pharmacist Association (APhA) ${ }^{68}$ Specifically, APhA policy statements express support for educating pharmacists and student pharmacists regarding the appropriate use of overdose reversal agents, the passage of federal and state laws that permit pharmacists to provide overdose reversal agents, and the role of pharmacists in directing and managing dosing, therapy, and education to patients regarding the appropriate and effective use of overdose reversal agents. ${ }^{69}$ Such support for naloxone education and distribution has been critical in the field as it recognizes that NMPO is an issue that has a serious impact on health professions across the continuum of care.

Complementary to naloxone overdose prevention, APhA policy also supports pharmacists taking on the role of health care providers by proactively screening and assessing patients' for possible NMPO and diversion. ${ }^{69} \mathrm{APhA}$ policy likewise promotes the need for a national PDMP to promote and sustain proactive clinical practices, such as universal 
patient monitoring. ${ }^{70}$ In the recent US White House meeting on addressing NMPO and the opioid overdose epidemic, APhA leadership pledged the development and publication of an online educational resource center for pharmacists and pharmacy technicians regarding NMPO. ${ }^{71}$ Such a resource for the pharmacy profession will potentially fill an important gap in information and has the capability of providing help for pharmacy professionals to more actively take on and address this issue. However, while developing such resources is an important aspect of a solution, APhA should dedicate resources to evaluate actual changes in practice behaviors of those professionals who utilize this online training resource.

The American Society of Health-System Pharmacists (ASHP) is likewise making significant efforts to address NMPO and overdose in the US. ASHP recommends that pharmacists proactively engage in prevention, education, and assistance to patients efforts to address NMPO and its consequences. ${ }^{72}$ Examples of prevention efforts recommended to health system pharmacists include collaborating with other health care professions to develop prevention programs, advocating and participating with public leaders to prevent NMPO, and working to discourage prescribing practices associated with behaviors indicative of abuse. ${ }^{72}$ Additionally, ASHP recommends that pharmacists provide information to patients and other health care professionals pertaining to substance use disorders, the effects of mood altering drugs, and safe storage/disposal of medications. Recommendations for educational efforts likewise include efforts to develop curricula for delivery in pharmacy training programs and university settings to assist the next generation of pharmacists to appropriately confront this issue. ${ }^{72}$ In terms of assistance to patients, ASHP encourages the active identification of patients engaged in NMPO and providing necessary referrals to treatment. Additionally, ASHP directs health-system pharmacists to remain vigilant in efforts to be gatekeepers of medications that may be the target of diversion or abuse. ${ }^{72}$ It comes as no surprise that ASHP was also a key contributor to federal efforts in strategizing the national response to NMPO and overdose by committing to the education of the organization's members on this issue. ${ }^{71}$

\section{Education and training programs}

Previous research has indicated that pharmacists who feel awkward or unconfident in their abilities to communicate with patients about NMPO are less likely to screen patients. ${ }^{73,74}$ Furthermore, pharmacists who report having little training in NMPO are less likely to discuss misuse with patients. ${ }^{74,75}$ Research has also shown that communicative, task-specific-efficacy beliefs, and participation in NMPO specific continuing education are significantly associated with addiction treatment information provision to pharmacy patients. ${ }^{76}$ Based on these facts, it is critical that student pharmacists and pharmacists participate in education, training, continuing education, and/ or board certification relevant to substance use disorders, risk minimization with opioid therapy, law update and overview of prescription drug abuse, drug diversion, new drugs of abuse, and pain management. Such training has the capacity to advance pharmacists' active involvement in prevention, education, and assistance of patients engaged in NMPO and/ or who suffer from other substance use disorders. Pharmacy schools and colleges must take the opportunity to further integrate education and practice opportunities around substance use disorders. ${ }^{76,77}$

Pharmacists are trained during their didactic curriculum and experiential learning to identify appropriate pharmacotherapy for patients, assess medication use outcomes, and ensure the safety and effectiveness of the medication-use system. During their education, student pharmacists are introduced to substance use disorders. Although each pharmacy school has a unique curriculum, the Accreditation Council for Pharmacy Education requires specific elements of the didactic doctor of pharmacy curriculum that are viewed as central to a contemporary high-quality pharmacy education, which includes both pharmacology and toxicology. ${ }^{78}$ Pharmacology introduces the knowledge of pharmacodynamics, which involves how drugs work in the body, mechanisms of therapeutic and adverse drug reactions and interactions, and the application of these principles to therapeutic decision making. Toxicology expands this medication knowledge by educating students on pharmacodynamics, mechanisms, prevention, and treatment of the toxic effects of drugs. Student pharmacists in many programs in the US have the opportunity to take elective coursework concerning addiction, wellness and prevention, as well as advanced pharmacy practice experiences utilizing community substance use disorder resources. ${ }^{77}$ Given the importance of training pharmacists regarding substance use disorders, the American Association of Colleges of Pharmacy has proposed curricular guidelines on substance use disorders that would allow future pharmacists entering the profession to have the training and ability to effectively pursue a role in substance abuse. ${ }^{77}$ With this breadth of knowledge, pharmacists entering practice have the education and training to serve in leadership and service roles in substance use disorder prevention and education and the ability to assist in a variety of patient care, employee health, and community activities involving addiction. ${ }^{78}$

Beyond the doctor of pharmacy education, pharmacists and student pharmacists have resources to aid in their 
education and training on NMPO and substance use disorders. One significant opportunity is provided by the $\mathrm{APhA}$ foundation in collaboration with the Ohio State University College of Pharmacy and the Cardinal Health Foundation. These entities have developed the Generation Rx program, an educational program that increases public awareness of NMPO and encourages health care providers, community leaders, parents, teens, and college students to actively work toward prevention. ${ }^{79}$ Generation $\mathrm{Rx}$ strives to increase awareness among pharmacists and student pharmacists with respect to the opportunity to serve as educators and to provide information and resources regarding the prevention of prescription medication abuse, delivery of materials necessary to effectively implement prescription abuse programs, and promotion of the pharmacy profession and its role in community health. ${ }^{80}$ Generation Rx similarly provides pharmacists with materials and knowledge to address substance abuse issues in a variety of areas of practice; including educational settings, among patients across the lifespan, and within the workplace. ${ }^{80}$

An additional training opportunity on addiction is provided through the APhA Institute on Alcoholism and Drug Dependencies. The institute has a long-standing history of providing education to pharmacists and student pharmacists regarding substance use disorders. The annual institute is delivered via seminar, wherein participants receive information regarding addiction and obtain practical experience in managing substance use disorder-related problems as they impact the pharmacy profession. Seminars follow a disease model pedagogy by explaining the pharmacology and pathophysiology of addiction, providing training with respect to the design of intervention strategies and techniques to provide care to patients in need, and aiding in the development of pharmacy-based strategies to assist patients to maintain substance use recovery. ${ }^{78}$

\section{Behavioral efforts}

As gaps in substance abuse training programs are identified, education and resources can further be developed to allow pharmacists to incorporate patient care and patient outcomes surrounding substance use disorders into practice. One immediate opportunity for pharmacists to address NMPO exists within community pharmacy practice. In the US, community pharmacies are abundant, with $>60,000$ community pharmacies, employing $>170,000$ pharmacists. ${ }^{81}$ Until the recent past, the dominant role of the community pharmacist has been in dispensing medications. However, the role of the pharmacist has been rapidly expanding to include a patient-centered focus and patient-centered outcomes. Central among the activities of this evolving focus are health education/promotion, supporting patients in achieving optimal medication adherence, and screening and monitoring patient health. ${ }^{82}$ Given these trends, pharmacists are continually increasing patient engagement and adapting their practice environments to meet the various needs of their patients. An innovative example of this is community pharmacies developing and utilizing private rooms for consultation in order to interact one-on-one regarding sensitive matters pertaining to the patient's heath. ${ }^{83-85}$

Such adaptation and evolution creates a distinct opportunity for community pharmacies to become a venue for the identification of patients at-risk or engaged in NMPO. Foundational clinical evidence has established that screening patients in community pharmacy for the risk of developing and/or current NMPO appears to have strong feasibility. ${ }^{86}$ Recent findings show that screening patients filling opioid medications with a computer tablet-based health screening survey was not only acceptable to patients, but such a protocol is not disruptive to pharmacy workflow as patients respond to screening questionnaires while they wait for medications to be processed and filled. ${ }^{86}$ Furthermore, the majority of patients $(\sim 80 \%)$ reported being comfortable if pharmacists engaged them in conversations about their pain medication consumption if pharmacists had any specific concerns. ${ }^{86}$

Unfortunately, there is a gap within the field of pharmacy with respect to evidence-based patient care services that have been tested and have demonstrated efficacy and/ or effectiveness for NMPO. The protocols that do exist in the field either are not opioid medication specific or have not been tested to this point in a clinical setting. In terms of existing models that are not opioid medication specific, MTM is an evidence-based intervention that can be delivered in community pharmacy setting. MTM involves brief sessions wherein medications are reviewed by pharmacists to assist patients resolve challenges related to adherence. A core goal of MTM is to empower patients in the active management of their medications. ${ }^{87,88}$ MTM services are currently considered an evidence-based practice that are reimbursed by Medicare and some private insurers. ${ }^{89}$ Other modalities employed in the field are motivational interviewing-based interventions. These approaches train pharmacists and other health care professionals to provide brief interventions that focus on helping patients increase their adherence to various medications. ${ }^{90-94}$ Models, such as MTM and motivational interviewing-based interventions, have many important components that possess the capacity for helping patients with NMPO.

In terms of protocols that have yet not been tested within clinical settings, a recently published model within the pharmacy practice literature is designed to address NMPO and more severe levels of opioid addiction that may present at 
community pharmacy settings. ${ }^{45}$ This model recommends that within the community pharmacy setting, all patients filling opioid pain medications be screened for NMPO and other health problems that would increase patients' risk for NMPO, such as mental, behavioral, and physical health conditions. For those who do not screen positive for NMPO or for other health risk factors, pharmacists are encouraged to continue to follow-up with those individuals during subsequent prescription fill visits in order to continuously monitor patient health. ${ }^{45}$ For patients who screen positive for conditions that increase their risk for developing NMPO, the model instructs that within an MTM framework, patients receive information and prevention messaging. ${ }^{45}$ Similarly, patients with health risks that increase chances for future NMPO would be referred back to their primary care physician or health insurance plans to initiate interdisciplinary care to ensure the conditions that increase risk for NMPO are properly managed/treated. For patients who screen positive for NMPO or more severe levels of opioid addiction, these individuals would receive brief adherence interventions and be trained by pharmacists in naloxone administration for fatal overdose prevention. ${ }^{45}$ Those patients with more severe levels of addiction would also receive referrals to primary or specialty care to be assessed for the need of agonist maintenance therapy. ${ }^{45}$

Currently, results of a pharmacist-led patient care protocol for NMPO have not been reported in the empirical literature. It is, therefore, paramount that clinical pharmacists and health services researchers collaborate to design and execute such studies. Information generated by these endeavors has the capacity to be transformative to professional pharmacy practice in how patients with NMPO or opioid medication addiction are treated within community pharmacy settings.

\section{Conclusion}

NMPO and opioid medication overdose are complex national public health issues that require multifaceted and broad-based efforts to effectively achieve appropriate management of access to these medications while providing patients with effective pain management. ${ }^{95}$ A number of pharmacy-based and related efforts have taken place in the US to confront this issue. National organizations that represent pharmacy practitioners have undertaken important efforts to increase pharmacist presence and influence among other health and governmental entities working toward resolution of NMPO, and pharmacy training and education programs have greatly augmented their efforts to equip those currently practicing and the next generation of pharmacists to effectively deal with this issue. Significant opportunities are available to pharmacists working in the community setting to identify patients with NMPO or with heighted risk for overdose; nevertheless, additional research must take place in order to establish evidence-based patient care protocols for national dissemination. As pharmacists and the pharmacy profession continue to lead in resolving this national issue, patient and public health will be improved.

\section{Disclosure}

The authors report no conflicts of interest in this work.

\section{References}

1. Office of National Drug Control Policy. Epidemic: Responding to America's Prescription Drug Abuse Crisis. Washington, DC: Office of National Drug Control Policy, Executive Office of the President of the United States; 2011.

2. Cochran G, Woo B, Lo-Ciganic W, Gordon AJ, Donohue J, Gellad W. Defining non-medical use of prescription opioids within health care claims: a systematic review. Subst Abus. 2015;36(2):192-202.

3. Smith SM, Dart RC, Katz NP, et al. Classification and definition of misuse, abuse, and related events in clinical trials: ACTTION systematic review and recommendations. Pain. 2013;154(11):2287-2296.

4. United Nations Office on Drugs and Crime. World Drug Report 2012. Vienna,Austria:United Nations Office on Drugs and Crime,United Nations; 2012.

5. International Narcotics Control Board, United Nations. Narcotic Drugs: Report 2013 Estimated World Requirements for 2014 - Statistics for 2012 Vienna,Austria:United Nations: International Narcotics Control_Board;2013.

6. Manchikanti L. National drug control policy and prescription drug abuse: facts and fallacies. Pain Physician. 2007;10(3):399-424.

7. Manchikanti L, Fellows B, Ailinani H, Pampati V. Therapeutic use, abuse, and nonmedical use of opioids: a ten-yearperspective. Pain Physician. 2010; 12(5):401-435.

8. Hanks S. The law of unintended consequences: when pain management leads to medication errors. P T. 2008;33(7):420-425.

9. Van Zee A. The promotion and marketing of OxyContin: commercial triumph, public health tragedy. Am J Public Health. 2009;99(2):221-227.

10. Comer SD, Sullivan MA, Whittington RA, Vosburg SK, Kowalczyk WJ. Abuse liability of prescription opioids compared to heroin in morphine-maintained heroin abusers. Neuropsychopharmacology. 2008; 33(5):1179-1191.

11. World Health Organization. Guidelines for the Psychosocially Assisted Pharmacological Treatment of Opioid Dependence. Geneva, Switzerland: World Health Organization; 2009.

12. Substance Abuse and Mental Health Services Administration. Results from the 2012 National Survey on Drug Use and Health: Summary of National Findings. Rockville, MD: Substance Abuse and Mental Health Services Administration; 2013.

13. Meyer R, Patel AM, Rattana SK, Quock TP, Mody SH. Prescription opioid abuse: a literature review of the clinical and economic burden in the United States. Popul Health Manag. 2014;17(6):372-387.

14. Cepeda MS, Fife D, Chow W, Mastrogiovanni G, Henderson SC. Assessing opioid shopping behaviour: a large cohort study from a medication dispensing database in the US. Drug Saf. 2012;35(4):325-334.

15. Hall AJ, Logan JE, Toblin RL, et al. Patterns of abuse among unintentional pharmaceutical overdose fatalities. JAMA. 2008;300(22):2613-2620.

16. Muhuri P, Gfroerer J, Davies C. Associations of Nonmedical Pain Reliever Use and Initiation of Heroin Use in the United States. Rockville, MD: Center for Behavioral Health Statistics and Quality, Substance Abuse and Mental Health Services Administration; 2013.

17. Compton WM, Jones CM, Baldwin GT. Relationship between nonmedical prescription-opioid use and heroin use. NEngl JMed.2016;374(2):154-163.

18. Cicero TJ, Ellis MS, Surratt HL, Kurtz SP. The changing face of heroin use in the United States: a retrospective analysis of the past 50 years. JAMA Psychiatry. 2014;71(7):821-826. 
19. White AG, Birnbaum HG, Schiller M, Tang J, Katz NP. Analytic models to identify patients at risk for prescription opioid abuse. Am J Manag Care. 2009;15(12):897-906.

20. Sullivan MD, Edlund MJ, Fan M-Y, Devries A, Brennan Braden J, Martin BC. Risks for possible and probable opioid misuse among recipients of chronic opioid therapy in commercial and Medicaid insurance plans: the TROUP Study. Pain. 2010;150(2):332-339.

21. Becker WC, Sullivan LE, Tetrault JM, Desai RA, Fiellin DA. Nonmedical use, abuse and dependence on prescription opioids among U.S. adults: psychiatric, medical and substance use correlates. Drug Alcohol Depend. 2008;94(1-3):38-47.

22. Amari E, Rehm J, Goldner E, Fischer B. Nonmedical prescription opioid use and mental health and pain comorbidities: a narrative review. Can J Psychiatry. 2011;56(8):495-502.

23. Huang B, Dawson DA, Stinson FS, et al. Prevalence, correlates, and comorbidity of nonmedical prescription drug use and drug use disorders in the United States: results of the National Epidemiologic Survey on Alcohol and Related Conditions. J Clin Psychiatry. 2006;67(7):1062-1073.

24. Martins SS, Keyes KM, Storr CL, Zhu H, Chilcoat HD. Pathways between nonmedical opioid use/dependence and psychiatric disorders: results from the National Epidemiologic Survey on Alcohol and Related Conditions. Drug Alcohol Depend. 2009;103(1-2):16-24.

25. Novak SP,Herman-Stahl M, Flannery B,Zimmerman M. Physical pain, common psychiatric and substance use disorders, and the non-medical use of prescription analgesics in the United States. Drug Alcohol Depend. 2009;100(1-2):63-70.

26. Rice JB, White AG, Birnbaum HG, Schiller M, Brown DA, Roland CL. A model to identify patients at risk for prescription opioid abuse, dependence, and misuse. Pain Med. 2012;13(9):1162-1173.

27. Unick GJ, Rosenblum D, Mars S, Ciccarone D. Intertwined epidemics: national demographic trends in hospitalizations for heroin- and opioidrelated overdoses, 1993-2009. PLoS One. 2013;8(2):e54496-e54496.

28. Braker LS, Reese AE, Card RO, Van Howe RS. Screening for potential prescription opioid misuse in a Michigan Medicaid population. Fam Med. 2009;41(10):729.

29. Hudson TJ, Edlund MJ, Steffick DE, Tripathi SP, Sullivan MD. Epidemiology of regular prescribed opioid use: results from a national, population-based survey. J Pain Symptom Manage. 2008;36(3):280-288.

30. Edlund MJ, Martin BC, Fan M-Y, Braden JB, Devries A, Sullivan MD. An analysis of heavy utilizers of opioids for chronic noncancer pain in the TROUP study. J Pain Symptom Manage. 2010;40(2):279-289.

31. Benyamin R, Trescot A, Datta S, et al. Opioid complications and side effects. Pain Physician. 2008;11(2 suppl):S105-S120.

32. Upadhyay J, Maleki N, Potter J, et al. Alterations in brain structure and functional connectivity in prescription opioid-dependent patients. Brain. 2010;133(pt 7):2098-2114.

33. Centers for Disease Control and Prevention. Vital Signs: Overdoses of Prescription Opioid Pain Relievers - United States, 1999-2008. Atlanta, GA: Centers for Disease Control and Prevention; 2011.

34. Centers for Disease Control and Prevention [webpage on the Internet]. Prescription Drug Overdose Data: Deaths from Prescription Opioid Overdose. 2015. Available from: http://www.cdc.gov/drugoverdose/ data/overdose.html. Accessed July 16, 2015.

35. Jones CM, Mack KA, Paulozzi LJ. Pharmaceutical overdose deaths, United States, 2010. JAMA. 2013;309(7):657-659.

36. Drug Enforcement Administration. 2014 National Drug Threat Assessment Summary. Ft. Bliss, TX: Drug Enforcement Administration Strategic Intelligence Section; 2014.

37. King SA. Preventing prescription opioid abuse: new formulations - but who will benefit? Psychiatr Times. 2009;26(2):16.

38. Severtson SG, Bartelson BB, Davis JM, et al. Reduced abuse, therapeutic errors, and diversion following reformulation of extended-release oxycodone in 2010. J Pain. 2013;14(10):1122-1130.

39. Rosenblatt RA, Andrilla CH, Catlin M, Larson EH. Geographic and specialty distribution of US physicians trained to treat opioid use disorder. Ann Fam Med. 2015;13(1):23-26.

40. Clark T, Eadie J, Knue P, Kreiner P, Strickler G. Prescription Drug Monitoring Programs: An Assessment of the Evidence for Best Practices.Waltham, MA: Heller School for Social Policy and Management, Brandeis University; 2012.
41. Prescription Drug Monitoring Program Center of Excellence. Briefing on PDMP Effectiveness. Waltham, MA: Prescription Drug Monitoring Program Center of Excellence at Brandeis; 2013.

42. Paulozzi LJ, Kilbourne EM, Desai HA. Prescription drug monitoring programs and death rates from drug overdose. Pain Med. 2011;12(5): $747-754$.

43. Shepherd J. Combating the prescription painkiller epidemic: a national prescription drug reporting program. Am J Law Med. 2014;40(1): $85-112$.

44. Perrone J, Nelson LS. Medication reconciliation for controlled substances - an "ideal" prescription-drug monitoring program. $N$ Engl J Med. 2012;366(25):2341-2343.

45. Cochran G, Gordon AJ, Field C, et al. Developing a framework of care for opioid medication misuse in community pharmacy. Res Social Adm Pharm. 2016;12(2):293-301.

46. Crozier MK, McMillan S, Hudson S, Jones S. The eastern North Carolina opioid prescribers project: a model continuing medical education workshop. J Opioid Manag. 2010;6(5):359-364.

47. Islam MM, McRae IS. An inevitable wave of prescription drug monitoring programs in the context of prescription opioids: pros, cons and tensions. BMC Pharmacol Toxicol. 2014;15:46.

48. Pringle JL, Boyer A, Conklin MH, McCullough JW, Aldridge A. The Pennsylvania Project: pharmacist intervention improved medication adherence and reduced health care costs. Health Aff. 2014;33(8): $1444-1452$

49. Khan NS, Norman IJ, Dhital R, McCrone P, Milligan P, Whittlesea $\mathrm{CM}$. Alcohol brief intervention in community pharmacies: a feasibility study of outcomes and customer experiences. Int J Clin Pharm. 2013; 35(6):1178-1187.

50. Bacci JL, McGrath SH, Pringle JL, Maguire MA, McGivney MS. Implementation of targeted medication adherence interventions within a community chain pharmacy practice: the Pennsylvania Project. $J \mathrm{Am}$ Pharm Assoc. 2014;54(6):584-593.

51. Cerulli J, Zeolla MM. Impact and feasibility of a community pharmacy bone mineral density screening and education program. J Am Pharm Assoc. 2004;44(2):161-167.

52. Lystlund S, Stevens E, Planas LG, Marcy TR. Patient participation in a clinic-based community pharmacy medication take-back program. $J$ Am Pharm Assoc. 2014;54(3):280-284.

53. Stewart H, Malinowski A, Ochs L, Jaramillo J, McCall K 3rd, Sullivan M. Inside Maine's Medicine Cabinet: Findings from the Drug Enforcement Administration's Medication Take-Back Events. Am J Public Health. 2015;105(1):e65-e71.

54. Thach AV, Brown CM, Pope N. Consumer perceptions about a community pharmacy-based medication take back program. J Environ Manage. 2013;127:23-27.

55. Welham GC, Mount JK, Gilson AM. Type and frequency of opioid pain medications returned for disposal. Drugs Real World Outcomes. 2015; 2(2):129-135.

56. Beletsky L, Rich JD, Walley AY. Prevention of fatal opioid overdose. JAMA. 2012;308(18):1863-1864.

57. Cheatle MD. Prescription opioid misuse, abuse, morbidity, and mortality: balancing effective pain management and safety. Pain Med. 2015; 16(suppl 1):S3-S8.

58. Kolodny A, Courtwright DT, Hwang CS, et al. The prescription opioid and heroin crisis: a public health approach to an epidemic of addiction. Annu Rev Public Health. 2015;36:559-574.

59. Green TC, Dauria EF, Bratberg J, Davis CS, Walley AY. Orienting patients to greater opioid safety: models of community pharmacy-based naloxone. Harm Reduct J. 2015;12:25.

60. American Association of Public Health [webpage on the Internet]. APHA Policy Statement LB-12-02 - Preventing Overdose Through Education and Naloxone Distribution. Washington, DC: 2016. Available from: https://www.apha.org/policies-and-advocacy/public-health-policystatements/policy-database/2014/07/16/13/08/reducing-opioid-overdose-through-education-and-naloxone-distribution\#. Accessed April 11, 2016.

61. Sporer KA.Acute heroin overdose. Ann Intern Med. 1999;130(7):584-590. 
62. Coffin PO, Fuller C, Vadnai L, Blaney S, Galea S, Vlahov D. Preliminary evidence of health care provider support for naloxone prescription as overdose fatality prevention strategy in New York City. J Urban Health. 2003; 80(2):288-290.

63. Wheeler E, Jones TS, Gilbert MK, Davidson PJ. Opioid overdose prevention programs providing naloxone to laypersons - United States, 2014. MMWR Morb Mortal Wkly Rep. 2015;64(23):631-635.

64. Walley AY, Xuan Z, Hackman HH, et al. Opioid overdose rates and implementation of overdose education and nasal naloxone distribution in Massachusetts: interrupted time series analysis. BMJ. 2013;346:f174.

65. Coffin PO, Sullivan SD. Cost-effectiveness of distributing naloxone to heroin users for lay overdose reversal. Ann Intern Med. 2013;158(1):1-9.

66. Bratberg J, Jackson A. Part II: law: educating and empowering patients and caregivers: the pharmacist's role in reducing the risk of opioid overdose. Drug Topics. 2016:51-65.

67. OkieS.Aflood of opioids, arising tide of deaths. NEnglJMed.2010;363(21): 1981-1985.

68. Robinson M [webpage on the Internet]. Combating Opioid Drug Abuse with Naloxone. 2015. Available from: https://www.pharmacist.com/ combating-opioid-drug-abuse-naloxone. Accessed Feburary, 2016.

69. American Pharmacists Association. Actions of the 2015 APhA House of Delegates. San Diego, CA: American Pharmacists Association; 2015.

70. American Pharmacists Association. American Pharmacists Association Policy Manual. Washington, DC: American Pharmacists Association; 2015.

71. Office of the Press Secretary. White House Obama Administration Announces Public and Private Sector Efforts to Address Prescription Drug Abuse and Heroin Use [Press Release]. Washington DC: Office of the Press Secretary; 2012.

72. American Society of Health-System Pharmacists. American Society of Health-System Pharmacists Statement on the Pharmacist's Role in Substance Abuse Prevention, Education, and Assistance. Washington, DC: American Society of Health-System Pharmacists; 2015.

73. Lafferty L, Hunter TS, Marsh WA. Knowledge, attitudes and practices of pharmacists concerning prescription drug abuse. J Psychoactive Drugs. 2006;38(3):229-232.

74. Cochran G, Field C, Lawson K. Pharmacists who screen and discuss opioid misuse with patients: future directions for research and practice. J Pharm Pract. 2015;28(4):404-412.

75. Wenthur CJ, Cross BS, Vernon VP, et al. Opinions and experiences of Indiana pharmacists and student pharmacists: the need for addiction and substance abuse education in the United States. Res Social Adm Pharm. 2013;9(1):90-100.

76. Hagemeier NE, Alamian A, Murawski MM, Pack RP. Factors associated with provision of addiction treatment information by community pharmacists. J Subst Abuse Treat. 2015;52:67-72.

77. American Association of Colleges of Pharmacy. Curricular Guidelines for Pharmacy: Substance Abuse and Addictive Disease. Alexandria, VA: American Association of Colleges of Pharmacy; 2010.

78. American Pharmacists Association [webpage on the Internet]. Institute on Alcoholism and Drug Dependencies. American Pharmacists Association 2016. 2016. Available from: http://www.pharmacist.com/apha-institute-alcoholism-and-drug-dependencies-program-objectives. Accessed February 22, 2016
79. American Pharmacists Association. Generation Rx [webpage on the Internet]. American Pharmacists Association Academy of Student Pharmacists. 2016. Available from: http://www.pharmacist.com/aphaasp-generation-rx. Accessed February 22, 2016.

80. GenerationRx [homepage on the Internet]. Generation Rx Safe Medication Practices for Life. 2016. Available from: http://www.generationrx.org/. Accessed February 21, 2016.

81. Centers for Disease Control and Prevention. Select Features of State Pharmacist Collaborative Practice Laws. Atlanta, GA: US Department of Health and Human Services, Centers for Disease Control and Prevention; 2013.

82. Fouche C, Butler R, Shaw J. Atypical alliances: the potential for social work and pharmacy collaborations in primary health care delivery. Soc Work Health Care. 2013;52(9):789-807.

83. Fallis J. A view from a room. Can Pharm J. 2012;145(6):254-255.

84. Mobach MP. The counter and consultation room work explored in the Netherlands. Pharm World Sci. 2008;30(4):360-366.

85. American Society of Health System Pharmacists. American Society of Health System Pharmacists Guidelines on Pharmacist-Conducted Patient Education and Counseling. Bethesda, MD: American Society of Health System Pharmacists; 1997.

86. Cochran G, Rubinstein J, Bacci JL, Ylioja T, Tarter R. Screening community pharmacy patients for risk of prescription opioid misuse. $J$ Addict Med. 2015;9(5):411-416.

87. American Pharmacist Associating, National Association of Chain Drug Stores Foundation. Medication therapy management in pharmacy practice: core elements of an MTM service model (version 2.0). J Am Pharm Assoc (2003). 2008;48:341-353.

88. Bluml BM. Definition of medication therapy management: development of professionwide consensus. JAm Pharm Assoc. 2005;45(5):566-572.

89. Perlroth D, Marrufo G, Montesinos A, et al. Medication Therapy Management in Chronically Ill Populations: Final Report. Burlingame, CA: Acumen, LLC; 2013.

90. Cook PF, Emiliozzi S, Waters C, El Hajj D. Effects of telephone counseling on antipsychotic adherence and emergency department utilization. Am J Manag Care. 2008;14(12):841-846.

91. Hill S, Kavookjian J. Motivational interviewing as a behavioral intervention to increase HAART adherence in patients who are HIVpositive: a systematic review of the literature. AIDS Care. 2012;24(5): $583-592$.

92. Taitel M, Jiang J, Rudkin K, Ewing S, Duncan I. The impact of pharmacist face-to-face counseling to improve medication adherence among patients initiating statin therapy. Patient Prefer Adherence. 2012;6: 323-329.

93. Kaplan JE, Keeley RD, Engel M, Emsermann C, Brody D. Aspects of patient and clinician language predict adherence to antidepressant medication. J Am Board Fam Med. 2013;26(4):409-420.

94. Barkhof E, Meijer CJ, de Sonneville LM, Linszen DH, de Haan L. The effect of motivational interviewing on medication adherence and hospitalization rates in nonadherent patients with multi-episode schizophrenia. Schizophr Bull. 2013;39(6):1242-1251.

95. Alford DP. Opioid prescribing for chronic pain - achieving the right balance through education. $N$ Engl J Med. 2016;374(4):301-303.
Integrated Pharmacy Research and Practice

\section{Publish your work in this journal}

Integrated Pharmacy Research and Practice is an international, peer-reviewed, open access, online journal, publishing original research, reports, reviews and commentaries on all areas of academic and professional pharmacy practice. This journal aims to represent the academic output of pharmacists and pharmacy practice with particular focus on integrated care. All papers are carefully
Dovepress

peer reviewed to ensure the highest standards as well as ensuring that we are informing and stimulating pharmaceutical professionals. The manuscript management system is completely online and includes a very quick and fair peer-review system, which is all easy to use. Visit http://www.dovepress.com/ testimonials.php to read real quotes from published authors. 